\title{
Tintinnids (Protozoa: Ciliophora) of the Büyükçekmece Bay in the Sea of Marmara*
}

\author{
NESLIHHAN BALKIS \\ Istanbul University, Faculty of Science, Department of Biology, 34459 Vezneciler-Istanbul, Turkey.
}

E-mail: neslbalk@istanbul.edu.tr

\begin{abstract}
SUMMARY: This study on the species composition and distribution of tintinnids, which form one of the most abundant ciliate groups in the seas, was carried out in Büyükçekmece Bay in 1998-1999. Plankton samples were collected horizontally from the subsurface $(0.5 \mathrm{~m})$ with a $55 \mu \mathrm{m}$ plankton net at five stations and a 31 water sampler was used for their quantitative analysis. All tintinnid species were identified according to their lorica shape as the usual important criterion. A total of 14 species were identified. Primary hydrographic conditions such as salinity, temperature and dissolved oxygen were recorded on each sampling occasion. The genera Favella and Eutintinnus were dominant in terms of species and individual numbers. Eutintinnus fraknoi was the most abundant species, with a mean abundance of $0.4 \times 10^{3}$ ind. $1^{-1}$. The study shows that the species and individual numbers of tintinnids decreased in winter and early spring when phytoplankton increases. The abundance of tintinnids in Büyükçekmece Bay appears to be correlated with the decrease in abundance of total phytoplankton $\left(r_{s}=-0.57, p=0.05\right)$ and diatoms $\left(r_{s}=-0.65, p<0.05\right)$ and the increase in temperature $\left(r_{s}=0.61, p<0.05\right)$. Furthermore, phytoplankton abundance was positively correlated to dissolved oxygen $\left(r_{s}=0.64, p<0.05\right)$. In particular, Favella serrata was more affected by temperature and Eutintinnus lusus-undae by salinity compared to other species.
\end{abstract}

Key words: tintinnids, abundance, phytoplankton, Büyükçekmece Bay, Sea of Marmara.

RESUMEN: TintínNidos (Protozoa: Ciliophora) de la bahía de BuyukCeKMece en el Mar de Mármara. - El presente estudio sobre la composición y distribución de las especies de tintínnidos, que forman parte de uno de los grupos más abundantes de ciliados marinos, fue realizado en la Bahía de Buyukcekmece en 1998-1999. Las muestras de plancton se recogieron horizontalmente a $0.5 \mathrm{~m}$ de la superficie con una red de plancton de $55 \mu \mathrm{m}$ en cinco estaciones y para el análisis cuantitativo se tomaban 3 litros de muestra. Todas las especies de tintínnidos eran identificadas de acuerdo a la forma de la lorica como principal criterio. Se identificó un total de 14 especies. Las condiciones hidrográficas como salinidad temperatura y oxigeno disuelto fueron determinadas en cada muestreo. El genero Favella y Eutintinnus dominaban en términos de abundancia. Eutintinnus frakmoi era la especie más abundante, con una media en abundancia de $0.4 \times 10^{3} \mathrm{ind} \mathrm{l}^{-1}$. El estudio muestra que el número de especies y el número de individuos por especie decrecía en verano y al principio de la primavera cuando el fitoplancton incrementa. La abundancia de tintínnidos en la Bahía de Buyukcekmece parece estar correlacionada con la disminución de la abundancia del fitoplancton total $\left(\mathrm{r}_{\mathrm{s}}=-0.57, \mathrm{p}=0.05\right)$ y diatomeas $\left(\mathrm{r}_{\mathrm{s}}=-0.65, \mathrm{p}<0.05\right)$ y con el incremento de temperatura $\left(r_{s}=0.61, p<0.05\right)$. Además, la abundancia de fitoplancton estaba positivamente correlacionada con la concentración de oxígeno disuelto $\left(\mathrm{r}_{\mathrm{s}}=0.64, \mathrm{p}<0.05\right)$. Concretamente Favella serrata estaba mas afectada por la temperatura y Eutintinnus lusus-undae por la salinidad comparado con otras especies.

Palabras clave: tintínnidos, abundancia, fitoplancton, bahía de Buyukcekmece, Mar de Mármara.

\section{INTRODUCTION}

Ciliates frequently dominate the microzooplankton and have a key position in planktonic food webs

*Received July 18, 2002. Accepted July 15, 2003. as they can respond quickly to phytoplankton pulses composed chiefly of nanoplankton (Capriulo and Ninivaggi, 1982). Tintinnids constitute one major component of marine planktonic ciliates and many species have an apparent cosmopolitan distribution in the seas and oceans (Marshall, 1969). 
The Büyükçekmece Bay is located in the northeast of the Sea of Marmara (Fig. 1). It remained connected to Büyükçekmece Lake until 1985, when the connection was blocked by a barrier $(11.4 \mathrm{~m}$ in height) in order to meet the need for fresh water in Istanbul (Meriç, 1986, 1992). Since then, the Büyükçekmece Dam Lake has had no effect on the dynamics of the bay because of lack of a water current from the lake to the bay (Meriç, 1992). No previous published studies exist on the tintinnids in the Sea of Marmara, a two-layer water body in which the surface water has characteristics of the Black Sea whereas the deep water has those of the Mediterranean Sea. A study by Sorokin et al. (1995) involving zooplankton reported that tintinnids were rare in the Sea of Marmara and mentioned the abundance of ciliates. There are few data on phytoplankton and their ecological features in the Sea of Marmara (Aubert et al., 1990; Uysal, 1996; Uysal and Ünsal, 1996; Balkıs, 2000, 2003). Of these, Balk1s (2003) presented data on seasonal variability and abundance of phytoplankton in Büyükçekmece Bay.

The aim of this study is to report on the biodiversity of tintinnids in surface waters in the Sea of Marmara and determine whether the occurrence of tintinnids is correlated to phytoplankton and selected hydrographical factors.

\section{MATERIAL AND METHODS}

This research was carried out in Büyükçekmece Bay. Tintinnids and phytoplankton samples for species identification were collected with horizontal tows from the subsurface $(0.5 \mathrm{~m})$ with a $55 \mu \mathrm{m}$ plankton net at five stations (Fig. 1) at monthly intervals from April 1998 to March 1999 and fixed in a $4 \%$ neutral formaldehyde solution. The $55-\mu \mathrm{m}$ net possibly underestimates the abundance of smaller tintinnids due to reduced retention. The species composition sampled with the plankton net should consequently be viewed as size-biased. Identification of smaller species was carried out using a 31 water sampler at a depth of $0.5 \mathrm{~m}$. Observations of the samples were made through the use of inverted phase contrast microscope equipped with a microphotosystem at a magnification of $400 \mathrm{X}$. For physical-chemical and quantitative analyses of tintinnid abundance, a 31 water sampler with thermometer was used at the same depth. These samples were preserved in acidified Lugol's iodine fixative (Throndsen, 1978). Fifty $\mathrm{ml}$ subsamples from 31 water sampler were concentrated by settling in special chambers for $24 \mathrm{~h}$ prior to analysis following the method of Utermöhl (Hasle, 1978) and counted. Also, the abundance of dominant phytoplankton

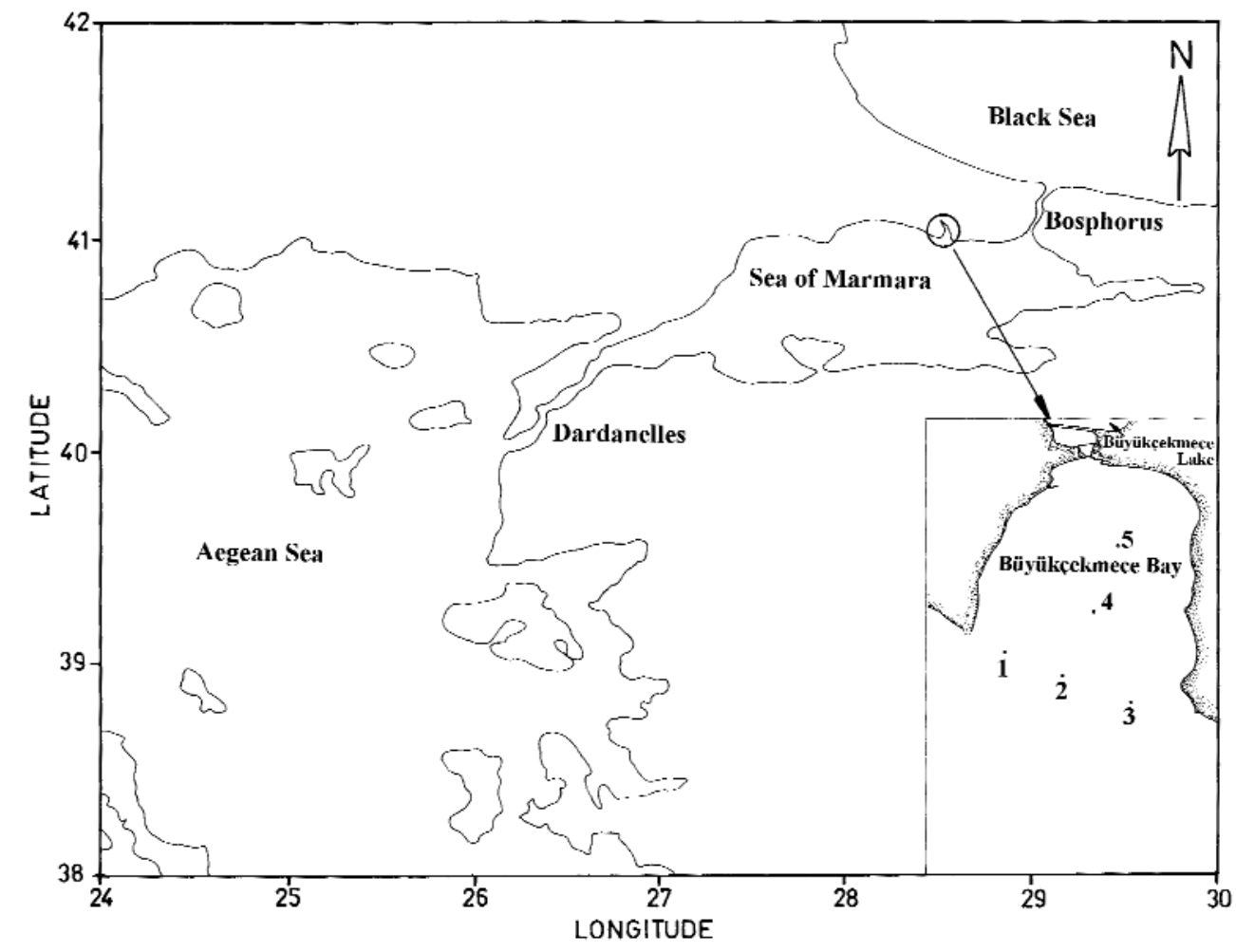

FIG. 1. - Research stations 1-5 in Büyükçekmece Bay. 
species was recorded. Phytoplankton samples were counted in a Sedgwick-Rafter cell using an inverted phase contrast microscope. Small forms of doubtful taxonomic classification were not added to the list and not counted (Table 1).

References used to identify the tintinnids and phytoplankton species were Lebour (1930), Cupp (1943), Trégouboff and Rose (1957), Balech (1959), Hendey (1964), Sournia (1968), Steidinger and Williams (1970), Drebes (1974), Taylor (1976), Dodge (1982), Koray and Özel (1983), Ricard (1987), Balech (1988), Hasle and Syvertsen (1997), Steidinger and Tangen (1997) and Throndsen (1997).

At each sampling date measurements of salinity (psu), temperature $\left({ }^{\circ} \mathrm{C}\right)$ and dissolved oxygen $(\mathrm{mg}$ $\mathrm{1}^{-1}$ ) were performed (Table 2). The Mohr-Knudsen method (Ivanoff, 1972) was used to measure salinity values, and the Winkler method (Winkler, 1888) to measure dissolved oxygen (DO) values. The abundance of tintinnids and phytoplankton and physico-chemical parameters of the five stations, where the study was carried out, are similar to one another and only the means for all stations are reported. Since the coefficient of variance $(\mathrm{V})$ calculated for five stations for each month was $<10 \%$ for temperature (0\%-5.8\%) and salinity (0.3\%-1.3\%), standard deviations (SD) were not given in the tables and figures. However, V (3.7\%-19\%) calculated for dissolved oxygen was $<10 \%$, with the exception of October-December, so the standard deviation for DO is given in Figure 2.

Spearman rank order correlation was used to correlate abundance of tintinnids with abundance of other phytoplanktonic organisms and hydrographical parameters. Moreover, Nonmetric Multi-Dimensional Scaling (MDS) analysis was performed to estimate relationships between the tintinnid community and hydrographic data.

\section{RESULTS}

\section{Abiotic parameters}

The average surface water temperature in the study area was $14.9^{\circ} \mathrm{C}$ (from $6.8 \pm 0.26^{\circ} \mathrm{C}$ to $23.5 \pm 0.35^{\circ} \mathrm{C}$ ). Salinity showed an average of 21.3 psu (from $19.7 \pm 0.25$ psu to $23.3 \pm 0.49 \mathrm{psu}$ ), and the average dissolved oxygen was $9.53 \mathrm{mg} \mathrm{l}^{-1}$ (from $7.13 \pm 1.37 \mathrm{mg} \mathrm{l}^{-1}$ to $11.95 \pm 0.44 \mathrm{mg} \mathrm{l}^{-1}$ ) (Fig. 2, Table 2). V calculated for DO was $>10 \%$ in October-

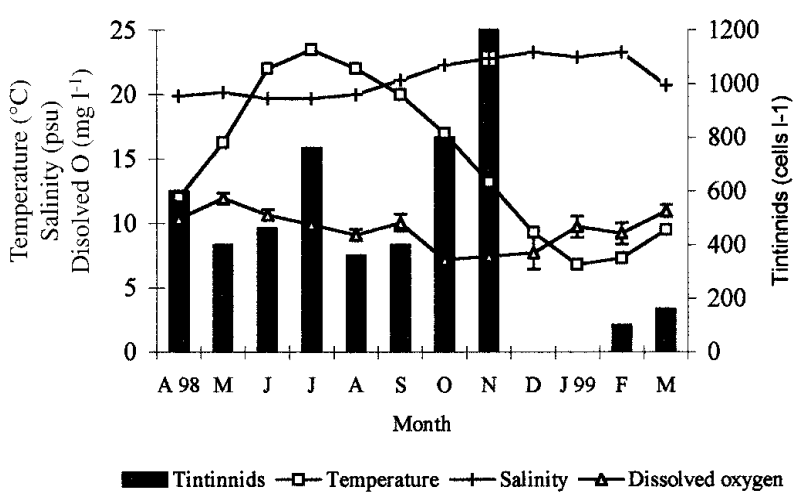

FIG. 2. - Monthly variations of abundance (cells $1^{-1}$ ) of total tintinnids, temperature $\left({ }^{\circ} \mathrm{C}\right)$, salinity (psu) and dissolved oxygen $\left(\mathrm{mg} \mathrm{l}^{-1}\right)$ in the surface water $(0.5 \mathrm{~m})$.

December only, the values being $19 \%(7.13 \pm 1.37$ $\left.\mathrm{mg} \mathrm{l}^{-1}\right), 17 \%\left(7.42 \pm 1.28 \mathrm{mg} \mathrm{l}^{-1}\right)$ and $16 \%(7.73 \pm 1.27$ $\left.\mathrm{mg} \mathrm{l}^{-1}\right)$ respectively.

\section{Tintinnid composition}

A total of 14 tintinnid species belonging to 9 genera and 5 families were identified (Table 1). Most of the tintinnids observed belong to the genera Favella and Eutintinnus. The latter was numerically the best represented genus. Total abundance of the tintinnid community varied greatly (Fig. 3). The maximum value for the period of this study was $1.2 \times 10^{3}$ ind. $\mathrm{l}^{-}$ ${ }^{1}$ in November. The lowest densities were observed in February and March, $0.1 \times 10^{3}$ and $1.6 \times 10^{2}$ ind. $1^{-1}$ respectively. In January and December no tintinnids were observed. Eutintinnus fraknoi was the most abundant species, with a mean abundance of $0.4 \times 10^{3}$ ind. $\mathrm{l}^{-1}$.

Tintinnid species were observed for ten months within a period of one year. Especially in October and November, it was found that tintinnids increased while the phytoplankton decreased. The abundance of tintinnids was $8 \times 10^{2}$ ind. $\mathrm{l}^{-1}$ in October and $1.2 \times 10^{3}$ ind. $1^{-1}$ in November. A. amphora, E. apertus, E. fraknoi, E. lusus-undae, F. serrata, H. subulata, $M$. jörgensenii and S. steenstrupii were the most abundant species. The highest species number was recorded in July (7 species) and November (6 species). The abundance of tintinnids was $7.6 \times 10^{2}$ ind. $1^{-1}$ in July. The species found during the sampling of this month were C. schabi, E. apertus, E. fraknoi, E. lusus-undae, F. campanula, F. ehrenbergi and T. radix. In February and March only one tintinnid species (F. ehrenbergi) was found (Table 1).

Generally, there was a negative correlation between the abundances of tintinnids and the record- 
TABLE 1. - The abundance (cells $1^{-1}$, average from 5 stations, see Fig. 1) and frequency (f) of each identified species from the subsurface (0.5 m) in Büyükçekmece Bay from April 1998 to March 1999. (Abbreviations used: V=very abundant, 81-100\%; A=abundant, 61-80\%; C= common, 41-60\%; R=rare, 21-40\%; $\mathrm{X}=$ present sporadically, 1-20\%).

\begin{tabular}{|c|c|c|c|c|c|c|c|c|c|c|c|c|c|}
\hline & $\begin{array}{c}\text { A } \\
1998\end{array}$ & M & $\mathrm{J}$ & $\mathrm{J}$ & A & S & $\mathrm{O}$ & $\mathrm{N}$ & $\mathrm{D}$ & $\begin{array}{c}\text { J } \\
1999\end{array}$ & $\mathrm{~F}$ & M & f \\
\hline \multicolumn{14}{|l|}{ Tintinnids } \\
\hline Amphorides amphora (Clap.and Lach.) Strand, 1926 & - & - & - & - & - & 60 & - & 160 & - & - & - & - & $\mathrm{X}$ \\
\hline Codonellopsis orthoceras (Haeckel) Jörgensen, 1924 & 140 & 200 & - & - & - & - & - & - & - & - & - & - & $\mathrm{X}$ \\
\hline C. schabi (Brandt) Kofoid and Campbell, 1929 & - & - & 100 & 100 & - & - & - & - & - & - & - & - & $\mathrm{X}$ \\
\hline Coxliella annulata (Daday) Brandt, 1907 & - & - & 220 & - & - & - & - & - & - & - & - & - & $\mathrm{X}$ \\
\hline Eutintinnus apertus Kofoid and Campbell, 1939 & - & - & - & 60 & 40 & - & - & 180 & - & - & - & - & $\mathrm{R}$ \\
\hline E. fraknoi (Daday) Kofoid and Campbell, 1939 & - & - & - & 100 & 100 & - & - & 400 & - & - & - & - & $\mathrm{R}$ \\
\hline E. lusus-undae (Entz) Kofoid and Campbell, 1939 & 360 & - & - & 40 & 100 & 160 & 160 & - & - & - & - & - & $\mathrm{C}$ \\
\hline Favella campanula (Schmidt) Jörgensen, 1924 & - & - & - & 200 & 80 & 60 & - & - & - & - & - & - & $\mathrm{R}$ \\
\hline F. ehrenbergi (Clap.and Lach.) Jörgensen, 1924 & 100 & - & 140 & 100 & - & - & - & - & - & - & 100 & 160 & $\mathrm{C}$ \\
\hline F.serrata (Mobius) Jörgensen, 1924 & - & 200 & - & - & - & 100 & 300 & - & - & - & - & - & $\mathrm{R}$ \\
\hline Helicostomella subulata (Ehren.) Jörgensen, 1924 & - & - & - & - & - & - & 100 & 160 & - & - & - & - & $\mathrm{X}$ \\
\hline Metacylis jörgensenii (Cleve) Kof. and Camp., 1929 & - & - & - & - & - & 20 & 140 & 220 & - & - & - & - & $\mathrm{R}$ \\
\hline Steenstrupiella steenstrupii (Clap. and Lach.) Kof. and Camp., 192 & $29-$ & - & - & - & - & - & 100 & 80 & - & - & - & - & $\mathrm{X}$ \\
\hline Tintinnopsis radix (Imhof) Brandt, 1907 & - & - & - & 160 & 40 & - & - & - & - & - & - & - & $\mathrm{X}$ \\
\hline Dinoflagellates & - & - & - & - & - & - & - & - & - & - & - & - & \\
\hline Ceratium furca (Ehrenberg) Clap. and & - & 990 & 290 & 600 & 200 & 140 & 140 & 430 & 110 & 40 & 70 & 60 & V \\
\hline C. fusus (Ehrenberg) Dujardin, 1841 & - & 170 & 140 & 870 & 1300 & 1100 & 1400 & 1500 & 30 & 90 & 210 & 590 & V \\
\hline C. minutum Jörgensen, 1920 & - & 20 & - & - & - & - & - & - & - & - & - & - & $\mathrm{X}$ \\
\hline C. trichoceros (Ehrenberg) Kofoid, 1908 & - & - & - & - & - & 130 & 130 & - & - & - & - & - & $\mathrm{X}$ \\
\hline C. tripos (O.F.Müller) Nitzsch, 1817 & 980 & 860 & 340 & 260 & 140 & - & - & - & - & - & - & 170 & $\mathrm{C}$ \\
\hline Dinophysis acuta Ehrenberg, 1839 & - & - & 10 & - & - & - & - & - & - & - & - & - & $\mathrm{X}$ \\
\hline D. caudata Saville-Kent, 1881 & - & - & - & - & - & - & - & 90 & 30 & - & - & - & $\mathrm{X}$ \\
\hline D. hastata Stein, 1883 & 190 & - & 10 & - & - & - & - & - & - & - & - & - & $\mathrm{X}$ \\
\hline Diplopsalis lenticula Bergh, 1881 & 7300 & 590 & 20 & - & 190 & 220 & - & - & - & 3000 & 1500 & 2900 & A \\
\hline Gonyaulax grindleyi Reinecke, 1967 & - & 200 & 40 & - & - & - & - & - & - & - & - & - & $\mathrm{X}$ \\
\hline G. monacantha Pavillard, 1916 & - & - & - & - & - & 70 & - & - & - & - & - & - & $\mathrm{X}$ \\
\hline Gymnodinium sanguineum Hirasaka, 1922 & 550 & 1340 & 440 & 290 & 100 & - & 30 & - & 50 & 570 & 60 & - & A \\
\hline G. simplex (Lohmann) Kofoi & 730 & 480 & - & - & - & - & - & - & - & - & - & - & $\mathrm{X}$ \\
\hline Heterocapsa triquetra (Ehrenberg) Stein, 1883 & - & 320 & - & - & - & - & - & - & - & - & 520 & - & $\mathrm{X}$ \\
\hline Kofoidinium velleloides Pavillard, 1928 & - & - & 40 & - & - & - & - & - & - & - & - & - & $\mathrm{X}$ \\
\hline Lingulodinium polyedrum (Stein) Dodge, 1989 & - & 650 & - & - & 140 & - & - & - & - & - & - & - & $\mathrm{X}$ \\
\hline Noctiluca scintillans (Macart.) Kof.and Swezy, 1921 & 190 & 210 & - & - & 30 & 20 & 30 & - & 30 & - & - & - & $\mathrm{C}$ \\
\hline Oxytoxum scolopax Stein, 1883 & - & - & - & - & - & - & 50 & - & 30 & - & - & - & $\mathrm{X}$ \\
\hline Phalacroma rotundatum (Clap.and Lach.) Kof. and Mich., 1911 & - & - & - & 20 & 120 & 60 & 60 & 40 & 160 & 80 & 80 & 60 & A \\
\hline Prorocentrum compressum (Bailey)Abe ex Dod., 1975 & 100 & - & 770 & 2500 & 4700 & - & 530 & 360 & 720 & 570 & - & - & A \\
\hline P. cordatum (Ostenfeld) Dodge, 1975 & - & - & & & & 530 & & & & & & - & $\mathrm{X}$ \\
\hline P. micans Ehrenberg, 1833 & 21001 & 18400 & 1200 & 3600 & 2800 & 6100 & 1500 & 1400 & 1900 & 620 & 1200 & 780 & $\mathrm{~V}$ \\
\hline P. scutellum Schröder, 1900 & 7303 & 32800 & 60 & 120 & 60 & 290 & 290 & 100 & 4900 & 3600 & 12100 & 1600 & $\mathrm{~V}$ \\
\hline P. triestinum Schiller, 1918 & 250 & - & - & 690 & 3700 & 6700 & 980 & - & 560 & - & - & - & $\mathrm{C}$ \\
\hline Protoperidinium bipes (Paulsen) Balech, 1974 & 1200 & 2100 & - & - & - & - & - & - & - & 490 & - & 60 & $\mathrm{R}$ \\
\hline P. brochi (Kofoid and Swezy) & - & - & - & - & - & - & 50 & - & - & - & - & - & $\mathrm{X}$ \\
\hline P. claudicans (Paulsen) Balech, 1974 & - & - & - & - & 30 & - & - & - & - & - & - & - & $\mathrm{X}$ \\
\hline P. crassipes (Kofoid) Balech, 1974 & - & - & 10 & 160 & - & - & - & - & - & - & & - & $\mathrm{X}$ \\
\hline P. depressum (Bailey) Balech, 1974 & 1400 & 250 & 30 & 30 & 30 & - & - & - & - & 840 & 760 & 500 & A \\
\hline P. divergens (Ehrenberg) Balech, 1974 & 250 & 800 & 290 & 360 & 90 & 150 & 270 & - & - & & & 170 & A \\
\hline P. leonis (Pavillard) Balech, 1974 & - & - & - & - & 110 & 150 & - & - & - & 210 & 260 & - & $\mathrm{R}$ \\
\hline P. paulseni Pavillard, 1905 & - & - & - & - & 210 & - & - & - & - & - & - & - & $\mathrm{X}$ \\
\hline P. pellucidum Bergh, 1881 & 3600 & 270 & - & - & - & - & - & - & - & - & 500 & 810 & $\mathrm{R}$ \\
\hline P. pentagonum (Gran) Balech, & - & 60 & - & - & - & - & - & - & - & - & - & - & $\mathrm{X}$ \\
\hline P. pyriforme (Paulsen) Balech, 1974 & - & 40 & - & - & 880 & 210 & - & - & - & - & 60 & - & $\mathrm{R}$ \\
\hline P. steinii (Jörgensen) Balech, 1974 & 230 & 210 & 140 & 180 & 180 & 30 & - & - & - & - & - & - & $\mathrm{C}$ \\
\hline P. subinerme (Paulsen) Loeblich III, 1970 & & - & - & - & - & 60 & - & - & - & - & - & - & $\mathrm{X}$ \\
\hline Protoperidinium spp. & 1400 & - & - & 170 & 380 & 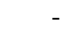 & - & - & - & - & - & - & $\mathrm{R}$ \\
\hline Pyrophacus horologium & & - & - & - & - & 30 & 100 & - & - & - & - & - & $\mathrm{X}$ \\
\hline Scrippsiella trochoidea (Stein) Loeblich III, 1976 & 1800 & 3100 & 650 & 830 & 840 & 870 & 40 & - & 60 & 4000 & 140 & 240 & V \\
\hline Diatoms & - & - & - & - & - & - & - & - & - & - & - & - & \\
\hline Achnanthes brevt & - & - & - & - & - & - & - & - & - & - & - & 60 & $\mathrm{X}$ \\
\hline Cerataulina pelagica (Cleve) Hendey, 1937 & - & - & 2700 & 740 & 3800 & - & - & 60 & 50 & - & - & - & $\mathrm{C}$ \\
\hline Chaetoceros spp. & 360 & 180 & 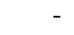 & 5100 & 2900 & 2000 & 180 & 1200 & 900 & 9500 & 2900 & 8400 & $\mathrm{~V}$ \\
\hline Climacosphenia spp. & & - & - & 130 & 30 & - & 60 & & & & & & $\mathrm{R}$ \\
\hline Coscinodiscus spp. & 470 & 510 & 260 & 30 & 710 & 50 & - & 110 & 150 & 170 & 150 & 240 & V \\
\hline Cylindroth & 120 & 20 & 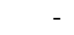 & 2700 & 150 & & 50 & 330 & 360 & 5700 & 24002 & 23100 & V \\
\hline Dactyliosolen fragilissimus (Bergon) Hasle, 1996 & - & - & - & - & - & 1100 & - & - & 2100 & - & 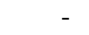 & - & $\mathrm{X}$ \\
\hline Ditylum brightwellii (West)Grun. in V.Heurck, 1883 & - & - & 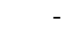 & - & - & - & 40 & 410 & 290 & 100 & 130 & - & $\mathrm{C}$ \\
\hline Guinardia flaccida (Castracane) Peragallo, 1892 & - & - & - & - & - & 270 & - & - & - & 280 & - & - & $\mathrm{X}$ \\
\hline Hemiaulus hauckii Grunow in Van Heurck, 1880-85 & - & - & - & - & & 3900 & 170 & 80 & - & - & - & - & $\mathrm{R}$ \\
\hline Leptocylindrus danicus Cleve, 1889 & - & - & 120 & - & 19001 & 15100 & - & - & - & 1500 & - & - & $\mathrm{R}$ \\
\hline Navicula spp. & 130 & 220 & - & - & - & - & - & - & - & - & 70 & 120 & $\mathrm{R}$ \\
\hline
\end{tabular}


TABLE 1 (Cont.). - The abundance (cells $1^{-1}$, average from 5 stations, see Fig. 1) and frequency (f) of each identified species from the subsurface $(0.5 \mathrm{~m})$ in Büyükçekmece Bay from April 1998 to March 1999. (Abbreviations used: V=very abundant, 81-100\%; A=abundant, 61-80\%; C= common, 41-60\%; $\mathrm{R}=$ rare, $21-40 \% ; \mathrm{X}=$ present sporadically, 1-20\%).

\begin{tabular}{|c|c|c|c|c|c|c|c|c|c|c|c|c|c|}
\hline & $\begin{array}{c}\text { A } \\
1998\end{array}$ & M & $\mathrm{J}$ & $\mathrm{J}$ & A & $\mathrm{S}$ & $\mathrm{O}$ & $\mathrm{N}$ & $\mathrm{D}$ & $\begin{array}{r}\mathrm{J} \\
199\end{array}$ & 9 & M & $\mathrm{f}$ \\
\hline Pleurosigma normanii Ralf in Pritchard, 1861 & - & - & 40 & - & - & 20 & - & - & - & - & - & - & $\mathrm{X}$ \\
\hline Proboscia alata (Brightwell) Sundsrôm, 1986 & - & 20 & - & - & 30 & 840 & - & - & - & - & - & - & $\mathrm{R}$ \\
\hline Pseudonitzschia spp. & - & - & - & - & 1100 & 1100 & 100 & 60 & 420 & 90 & - & 120 & $\mathrm{C}$ \\
\hline Pseudosolenia calcar-avis (Schult.) Sundsrôm, 1986 & - & - & 40 & 1600 & 1100 & 40 & - & 1100 & - & - & - & - & $\mathrm{C}$ \\
\hline Rhizosolenia setigera Brightwell, 1858 & - & - & - & - & - & - & - & - & 60 & 12300 & 10100 & 1800 & $\mathrm{R}$ \\
\hline Skeletonema costatum (Greville) Cleve, 1878 & 1900 & - & 90 & 2000 & 2900 & 350 & - & 230 & - & 1700 & 37002 & 250000 & A \\
\hline Striatella unipunctata (Lyngbye) Agardh, 1832 & - & - & 20 & - & - & - & - & - & - & - & - & - & $\mathrm{X}$ \\
\hline Thalassionema nitzschioides (Grunow)Meresh., 1902 & - & - & - & 50 & 1400 & - & - & - & - & - & 130 & - & $\mathrm{R}$ \\
\hline Thalassiosira anguste-lineata (Sch.) Fry.and Hasle, 1977 & - & - & - & - & - & - & - & - & - & 1300 & 5700 & 400 & $\mathrm{R}$ \\
\hline T. rotula Meunier, 1910 & - & - & - & - & - & - & - & - & 1100 & - & 1900 & - & $\mathrm{X}$ \\
\hline Silicoflagellates & - & - & - & - & - & - & - & - & - & - & - & - & \\
\hline Dictyocha fibula Ehrenberg, 1839 & - & - & - & 50 & 130 & 30 & - & - & 530 & 130 & - & - & $\mathrm{C}$ \\
\hline D. speculum Ehrenberg, 1839 & - & - & 20 & - & - & - & - & - & - & - & - & 60 & $\mathrm{X}$ \\
\hline Octactis octonaria (Ehrenberg) Hovasse, 1946 & - & - & 20 & - & - & - & - & - & - & - & - & - & $\mathrm{X}$ \\
\hline Euglenophycean & - & - & - & - & - & - & - & - & - & - & - & - & \\
\hline Eutreptiella spp. & - & - & - & - & & 59400 & - & - & - & - & - & - & $\mathrm{X}$ \\
\hline Prasinophycean & - & - & - & - & - & - & - & - & - & - & - & - & \\
\hline Halosphaera viridis Schmitz, 1878 & - & - & - & - & - & - & 50 & - & 1000 & - & - & - & $\mathrm{X}$ \\
\hline Tintinnids & 600 & 400 & 460 & 760 & 360 & 400 & 800 & 1200 & - & - & 100 & 160 & \\
\hline Dinoflagellates & 23000 & 63860 & 4480 & 10680 & 16230 & 16860 & 5600 & 3920 & 8580 & 14110 & 17460 & 7940 & \\
\hline Diatoms & 2980 & 950 & 3270 & 12350 & 16020 & 24770 & 600 & 3580 & 5430 & 32640 & 27180 & 284240 & \\
\hline Silicoflagellates & - & - & 40 & 50 & 130 & 30 & - & - & 530 & 130 & - & 60 & \\
\hline Euglenophycean & - & - & - & - & - & 59400 & - & - & - & - & - & - & \\
\hline Prasinophycean & - & - & - & - & - & - & 50 & - & 1000 & - & - & - & \\
\hline Total Phytoplankton & 25980 & 64810 & 7790 & 23080 & 32380 & 101060 & 6250 & 7500 & 15540 & 46880 & 44640 & 292240 & \\
\hline
\end{tabular}

ed phytoplankton (Fig. 3). Spring phytoplankton blooms generally developed and presumably persist because of low grazing pressure from zooplankton. Throughout the year, total phytoplankton reached its maximum values at a depth of $0.5 \mathrm{~m}\left(292 \times 10^{3}\right.$ ind. $\left.1^{-1}\right)$ in March. Diatoms in particular increased (284 $\mathrm{x} 10^{3}$ ind. $\mathrm{1}^{-1}$ ) and only the tintinnid $F$. ehrenbergi $\left(1.6 \times 10^{2}\right.$ ind. $\left.1^{-1}\right)$ was observed. S. costatum was the species with the most significant increase $\left(250 \times 10^{3}\right.$ ind. $\left.1^{-1}\right)$, followed by $C$. closterium $\left(23.1 \times 10^{3}\right.$ ind. $\left.1^{-1}\right)$ and Chaetoceros spp. $\left(8.4 \times 10^{3}\right.$ ind. $\left.1^{-1}\right)$ in that order. Only low numbers of dinoflagellates were recorded in this month, with the exception of Diplopsalis lenticula $\left(2.9 \times 10^{3}\right.$ ind. $\left.1^{-1}\right)$ and P. scutellum $\left(1.6 \times 10^{3}\right.$ ind. $\left.1^{-1}\right)$. In April when the dinoflagellates were numerically dominant $\left(23 \times 10^{3}\right.$ ind. $\left.1^{-1}\right)$, the number of tintinnids was $6 \times 10^{2}$ ind. $1^{-1}$. During this month only 3 tintinnid species were observed (C. orthoceras, E. lusus-undae, F. ehrenbergi). Among dinoflagellates and diatoms, the most copious were D. lenticula $\left(7.3 \times 10^{3}\right.$ ind. $\left.1^{-1}\right), P$. pellucidum $\left(3.6 \times 10^{3}\right.$ ind. $\left.1^{-1}\right)$, P. micans $\left(2.1 \times 10^{3}\right.$ ind. $\left.{ }^{-1}\right)$ and $S$. costatum $\left(1.9 \times 10^{3}\right.$ ind. $\left.1^{-1}\right)$. May was another important month in which total phytoplankton increased. $P$. scutellum $\left(33 \times 10^{3}\right.$ ind. $\left.1^{-1}\right)$ and $P$. micans $\left(18 \times 10^{3}\right.$ ind. $\left.1^{-1}\right)$ were present in large amounts at that depth. The species found during the sampling of this month were $C$. orthoceras $\left(2 \times 10^{2}\right.$ ind. $\left.1^{-1}\right)$ and $F$. serrata $\left(2 \times 10^{2}\right.$ ind. $\left.1^{-1}\right)$.

In the summer, total phytoplankton reached its maximum values in July $\left(23 \times 10^{3}\right.$ ind. $\left.\mathrm{I}^{-1}\right)$ and August $\left(32.4 \times 10^{3}\right.$ ind. $\left.1^{-1}\right)$. The most significant diatom species in July were Chaetoceros spp. $\left(5.1 \times 10^{3}\right.$ ind. $\left.1^{-1}\right)$, Cylindrotheca closterium $\left(2.7 \times 10^{3}\right.$ ind. $\left.1^{-1}\right)$, Skeletonema costatum $\left(2 \times 10^{3}\right.$ ind. $\left.1^{-1}\right)$ and Pseudosolenia calcar-avis $\left(1.6 \times 10^{3}\right.$ ind. $\left.1^{-1}\right)$ and the dinoflagellates P. micans $\left(3.6 \times 10^{3}\right.$ ind. $\left.1^{-1}\right)$ and Prorocentrum compressum $\left(2.5 \times 10^{3}\right.$ ind. $\left.1^{-1}\right)$. The high-

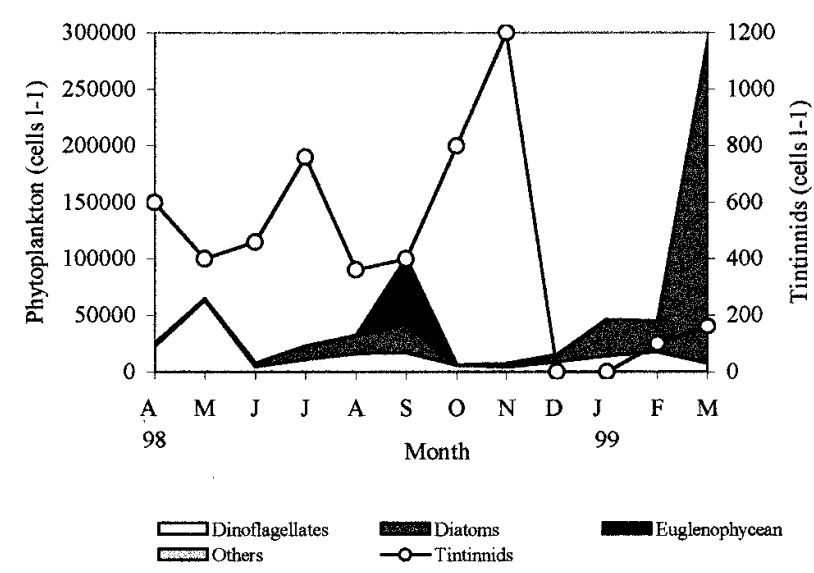

FIG. 3. - Monthly variations of abundance (cells $1^{-1}$ ) of total tintinnids and phytoplankton in the surface water. 
TABLE 2. - Tintinnid species obtained in Büyükçekmece Bay and some hydrographical parameters. Temperature: ${ }^{\circ} \mathrm{C}$; salinity: psu; dissolved oxygen: $\mathrm{mg}^{1-1}$.

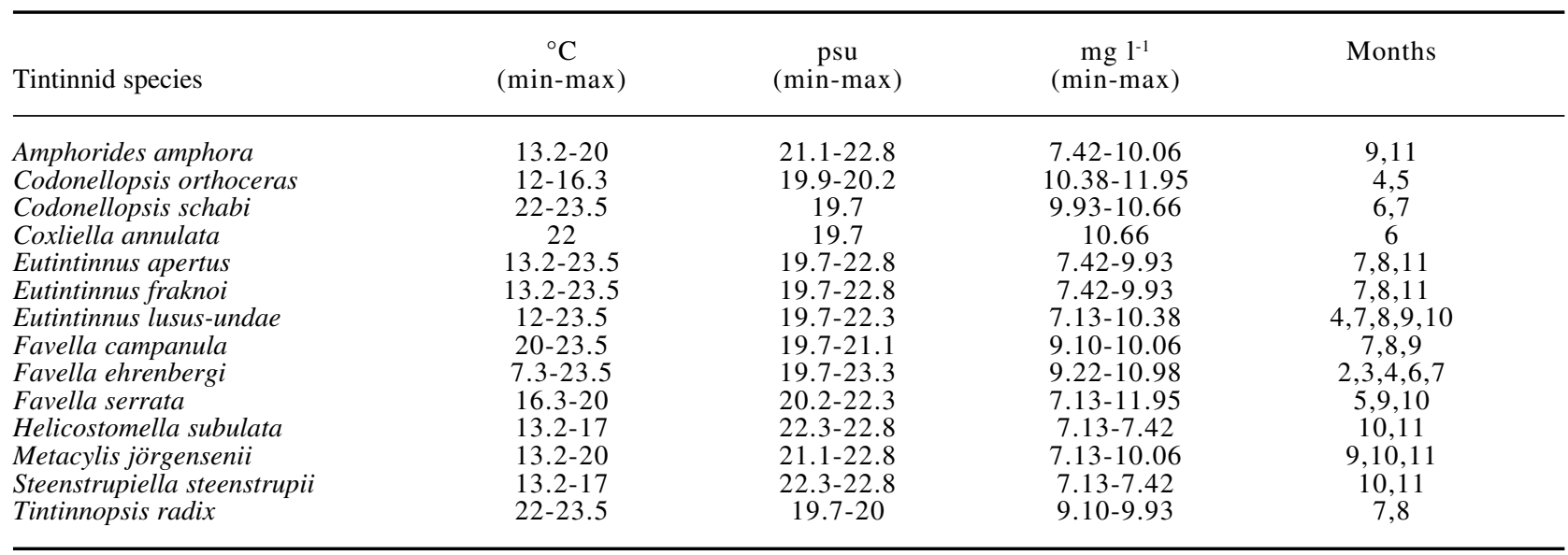

est tintinnid species number was recorded in this month (7 species). In August, the dinoflagellates $P$. compressum $\left(4.7 \times 10^{3}\right.$ ind. $\left.1^{-1}\right)$, P. triestinum $\left(3.7 \times 10^{3}\right.$ ind. $\left.\mathrm{1}^{-1}\right), P$. micans $\left(2.8 \times 10^{3}\right.$ ind. $\left.\mathrm{1}^{-1}\right)$ and $C$. fusus $\left(1.3 \times 10^{3}\right.$ ind. $\left.1^{-1}\right)$, and the diatoms $C$. pelagica

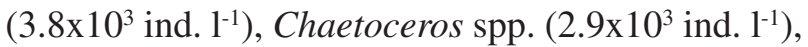
S. costatum $\left(2.9 \times 10^{3}\right.$ ind. $\left.{ }^{-1}\right)$, L. danicus $\left(1.9 \times 10^{3}\right.$ ind. $\left.\mathrm{l}^{-1}\right)$ and T. nitzschioides $\left(1.4 \times 10^{3}\right.$ ind. $\left.\mathrm{1}^{-1}\right)$ dominated.

In autumn, another phytoplankton peak was detected in September $\left(101 \times 10^{3}\right.$ ind. $\left.\mathrm{l}^{-1}\right)$, dominated by the Euglenophycean Eutreptiella spp. $\left(59.4 \times 10^{3}\right.$ ind. $\left.1^{-1}\right)$ and the diatom L. danicus $\left(15.1 \times 10^{3}\right.$ ind. $\left.1^{-1}\right)$. During this month five tintinnid species were observed (A. amphora, E. lusus-undae, F. campanu- la, $F$. serrata, M. jörgensenii). In October and November the dinoflagellates, Ceratium fusus and Prorocentrum micans, were dominant. The maximum cell number of $C$. fusus was $1.4 \times 10^{3}$ ind. $1^{-1}$ in October and $1.5 \times 10^{3}$ ind. $1^{-1}$ in November, whereas that of P. micans was $1.5 \times 10^{3}$ ind. $1^{-1}$ in October and $1.4 \times 10^{3}$ ind. $1^{-1}$ in November.

In winter, the number of diatoms increased more than it did in the other seasons. In January, the predominant species of diatoms were $R$. setigera $\left(12.3 \times 10^{3}\right.$ ind. $\left.1^{-1}\right)$, Chaetoceros spp. $\left(9.5 \times 10^{3}\right.$ ind. $\left.1^{-1}\right)$ and $C$. closterium $\left(5.7 \times 10^{3}\right.$ ind. $\left.1^{-1}\right)$. The tintinnid F. ehrenbergi was only observed in February in this season. In February, the dinoflagellate P. scutellum $\left(12.1 \times 10^{3}\right.$ ind. $\left.1^{-1}\right)$ and the diatoms $R$. setigera

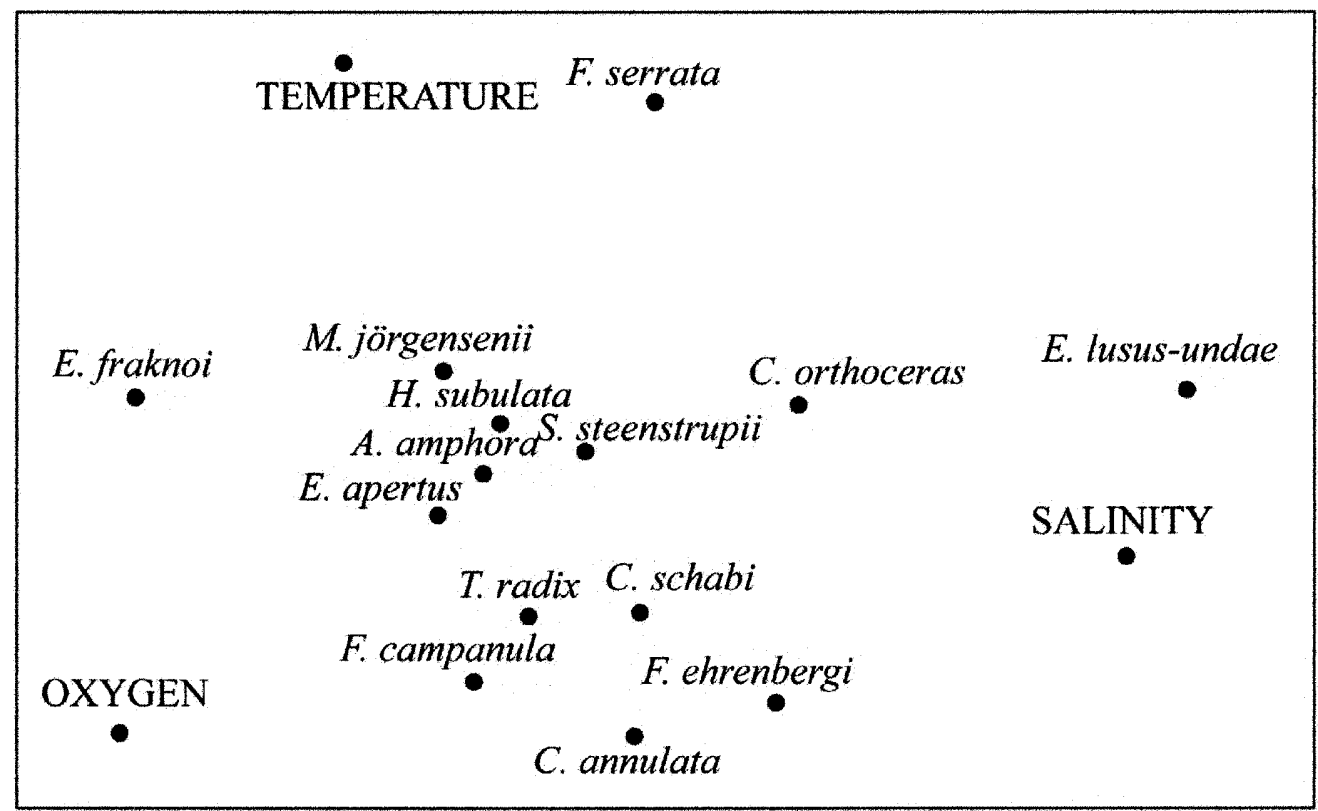

FIG. 4. - Multi-Dimensional Scaling (MDS) plot in two dimensions for the tintinnid community in Büyükçekmece Bay with relation to temperature, salinity and oxygen. 
$\left(10.1 \times 10^{3}\right.$ ind. $\left.1^{-1}\right)$, T. anguste-lineata $\left(5.7 \times 10^{3}\right.$ ind. $\left.1^{-1}\right)$ and $S$. costatum $\left(3.7 \times 10^{3}\right.$ ind. $\left.1^{-1}\right)$ dominated.

The generalist species that were found throughout the whole year were the dinoflagellates Prorocentrum micans $(\mathrm{f}=100 \%)$, P. scutellum $(\mathrm{f}=100 \%)$, Ceratium furca (f=92\%), C. fusus ( $\mathrm{f}=92 \%)$, Scrippsiella trochoidea $(\mathrm{f}=92 \%)$, Gymnodinium sanguineum ( $\mathrm{f}=75 \%)$ and Phalacroma rotundatum $(\mathrm{f}=75 \%)$, and the diatoms Chaetoceros spp. ( $\mathrm{f}=92 \%)$, Coscinodiscus spp. $(\mathrm{f}=92 \%)$, Cylindrotheca closterium $(\mathrm{f}=83 \%)$ and Skeletonema costatum $(\mathrm{f}=75 \%)$ (Table 1$)$.

The abundance of tintinnids in Büyükçekmece Bay appears to be negatively correlated to the abundance of total phytoplankton $\left(\mathrm{r}_{\mathrm{s}}=-0.57, \mathrm{p}=0.05\right)$ and diatoms $\left(r_{s}=-0.65, p<0.05\right)$ and positively correlated to temperature $\left(r_{s}=0.61, p<0.05\right)$. Furthermore, phytoplankton abundance is positively correlated to dissolved oxygen $\left(r_{s}=0.64, p<0.05\right)$. The other parameters did not appear to play any role in the dynamics of the plankton community of Büyükçekmece Bay. In particular, F. serrata was more affected by temperature and E. lusus-undae by salinity compared to other species (Fig. 4).

\section{DISCUSSION}

There are 90 ciliate species known to exist in all the seas of Turkey (Koray et al., 1999). Öztürk (1999) reported 17 whereas Türkoğlu and Koray (2000) reported 18 tintinnid species found in the Turkish territorial waters of the Black Sea. Off the coasts of Ukraine, Romania, Bulgaria and Georgia 27, 15, 23 and 9 tintinnid species were found respectively (Petranu, 1997; Zaitsev and Alexandrov, 1998; Konsulov, 1998; Komakhidze and Mazmanidi, 1998). This study reports on 14 tintinnid species found in the Sea of Marmara. All the species found in Büyükçekmece Bay are known to occur in the Aegean and the Mediterranean Seas, while only Coxliella annulata, Favella campanula, F. ehrenbergi, F. serrata, Helicostomella subulata and Tintinnopsis radix are present in the Black Sea. If the sampling had been carried out using a smaller mesh size, more tintinnid species might have been detected.

In this study of the surface waters of the Büyükçekmece Bay the highest numbers of tintinnid species were found in July and November and the lowest number in February and March. In January and December no tintinnids were observed. A nega- tive correlation was observed between tintinnids and the recorded phytoplankton species. In particular, tintinnids increased in both individual number and in species during October and November, when there was a decrease in phytoplankton. In March, in contrast to the increases in diatom abundance, the tintinnid abundance decreased. This may be explained by the general inability of ciliates to feed on colonial diatoms and large dinoflagellates (Hansen, 1991a). Ciliates mainly feed on nanosized prey, preferably nanoflagellates (Burkill et al., 1987; Dolan and Coats, 1990; Paranjape, 1990; Sherr and Sherr, 1994). It is possible that the nanoflagellates were abundant when the large dinoflagellates and diatoms were not, which would explain the negative correlation between tintinnids and the recorded phytoplankton species. Since nanoflagellate abundance was not measured in this study, the role of nanoflagellates remains unknown. However, Aubert et al. (1990) found that nanoflagellates were common in the Sea of Marmara in July $\left(1.3 \times 10^{6}\right.$ ind. $\left.1^{-1}\right)$. This value is concordant with the peak shown by tintinnids in the summer period. Nevertheless, Aubert et al. (1990) did not mention nanoflagellate abundance in November, when tintinnids appear to reach a maximum in this study.

Apart from nanoflagellates, there are several other causes for the negative correlations between tintinnid abundance and phytoplankton abundance. Also, heterotrophic and mixotrophic dinoflagellates are often numerous in marine plankton and are considered important consumers of both phytoplankton and bacteria (Hansen, 1991a; Bockstahler and Coats, 1993a), and they can consume ciliates (Bockstahler and Coats, 1993b). Mixotrophy appears to be widespread among prymnesiophytes and many dinoflagellates (Hansen and Nielsen, 1997; Hansen, 1998). G. sanguineum is one of several species of large mixotrophic dinoflagellates and a predator of ciliates (Bockstahler and Coats, 1993b). In this study, the highest cell number of G. sanguineum was found in May $\left(1.3 \times 10^{3}\right.$ ind. $\left.1^{-1}\right)$. Also, Dinophysis hastata and Phalacroma rotundatum can ingest ciliates (Hansen, 1991b), but these species did not reach great numbers during the sampling period. Other mixotrophic dinoflagellates such as Ceratium and Dinophysis, and heterotrophic ones such as Diplopsalis, Gymnodinium, Noctiluca and Protoperidinium were found throughout the year. However, the abundance was generally low and it is thus not likely that there was any major grazing pressure from dinoflagellates on ciliates. It is more likely that other preda- 

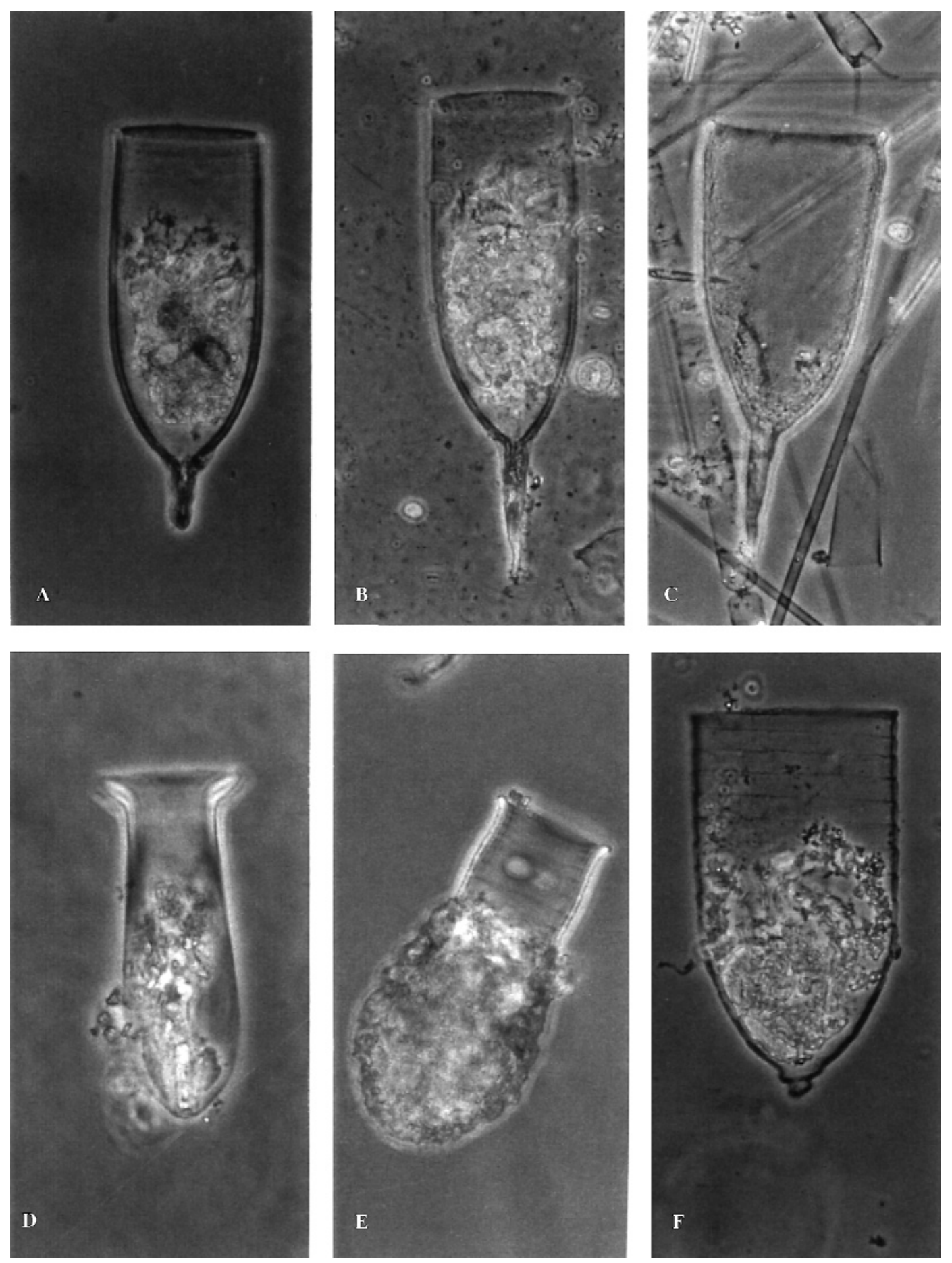

FIG. 5. - A, Favella campanula (x 200); B, F. ehrenbergi (x 200); C, F. serrata (x 200; D, Amphorides amphora (x 400); E, Codonellopsis schabi (x 400); F, Coxliella annulata (x 200).

tors, i.e. mesozooplankton, were more important consumers of the tintinnids (Turner and Anderson, 1983; Turner et al., 1998; Coats and Revalente, 1999; Levinsen and Nielsen, 2002). Since, mesozooplankton was not measured in this study, we do not know the extent of this predation, but it would be interesting to study it in the future since it could explain some of the seasonal patterns of the tintinnids that were found. Only Uysal (1996) reported individuals of different zooplankton groups formed by copepods, siphonophores, chaetognaths, polychaete larvae, cladocerans and appendicularians in the Sea of Marmara. The percentage distribution of zooplankton groups revealed that the predominance 

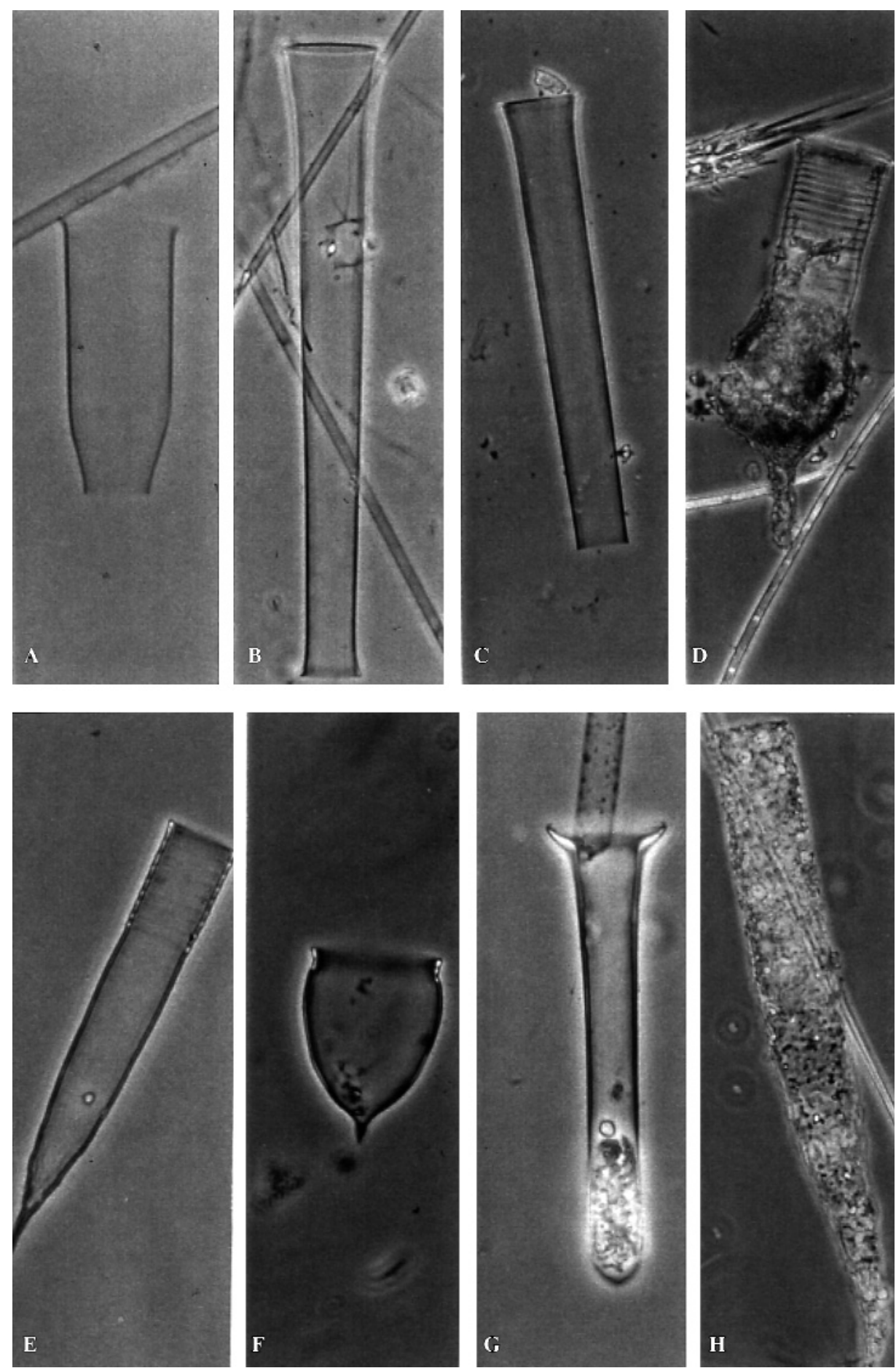

Fig. 6. - A, Eutintinnus apertus (x 400); B, E. fraknoi (x 200); C, E. lusus-undae (x 200); D, Codonellopsis orthoceras (x 200); E, Helicostomella subulata (x 400); F, Metacylis jörgensenii (x 400); G, Steenstrupiella steenstrupii (x 400); H, Tintinnopsis radix (x 200).

of copepods persists throughout the year in the region, and the highest recorded zooplankton level for the upper layer was $125400 \mathrm{ind} . / \mathrm{m}^{3}$ in September 1985 , and the lowest was $3980 \mathrm{ind} . / \mathrm{m}^{3}$.

Other factors that can cause the negative correlation between phytoplankton and ciliates include the possibility that some phytoplankton may produce chemical defence compounds. The best-known are toxin-producing dinoflagellates, which may have a negative impact on tintinnids (Hansen et al., 1992). A number of marine dinoflagellates have been known to produce nonprotein toxins, and these dinoflagellates are capable of forming red tides that inhibit zooplankton grazing (Hansen, 1989). During 
late spring/summer, peaks for phytoplankton abundances and production have been recorded in many Mediterranean coastal regions. Red tides are also more frequent at these times of the year (Zingone et al., 1990). In this study, none of the phytoplanktonic species exceeded one million cells per litre of surface water. At no time during this study was any colouring of the surface water detected. Despite the presence of certain dinoflagellate species (Ceratium furca, Dinophysis acuta, Heterocapsa triquetra, Lingulodinium polyedrum, Noctiluca scintillans, Phalacroma rotundatum, Prorocentrum micans, $P$. triestinum, Scrippsiella trochoidea) responsible for red tides and other noxious algal blooms in other geographic areas (Koray et al., 1992; Hallegraeff, 1993; Smalley and Coats, 2002), red tides were not recorded during the sampling period of this study.

Throughout the year, the tintinnid species were found in the range of $7.3-23.5^{\circ} \mathrm{C}, 19.7-23.3 \mathrm{psu}$ and 7.13-11.95 $\mathrm{mg} \mathrm{l}^{-1}$. These values are characteristic for this area (Ünlüata et al., 1990; Beşiktepe et al., 1995) and the chemical oceanography of the Sea of Marmara is significantly influenced by the biochemistry of the Black Sea and the Aegean Sea. It connects to the Black Sea through the Bosphorus in the NE and to the Aegean Sea via the Dardanelles in the SW. The basin is occupied by two distinctly different water masses throughout the year: the brackish waters (22-26 psu) of the Black Sea origin, forming a relatively thin surface layer (10-15 m thick) with a mean residence time of about 4-5 months, and the subhalocline waters of Mediterranean origin (38.5$38.6 \mathrm{psu}$ ) separated from the former by a sharp interface (pycnocline) about 10-20 m thick. Because of the large volume of water inflow from the adjacent Black Sea (about $600 \mathrm{~km}^{3}$ ) into the relatively small upper layer volume (about $225 \mathrm{~km}^{3}$ ) of the Sea of Marmara, the upper layer ecosystem of the latter has been influenced to a large extent. (Ünlüata et al., 1990; Tuğrul and Polat, 1995). In particular, at depths of $0.5-20 \mathrm{~m}$, the Sea of Marmara is known to be affected by the brackish water coming from the Black Sea via the Bosphorus (Yüce and Türker, 1991). The abundance of tintinnids has been effected by different water masses in the area. It was observed that the maximum abundance of tintinnids was found when salinity was high and temperature low. It is known that there are limited vertical exchanges between water masses due to thermocline and halocline layers, particularly during spring and summer, and the water on the surface does not usually sink down to the bottom. In autumn and winter, winds cause the water to become rough, the stratification is broken up, and the water from the bottom comes up to the surface (Balkis, 2003). Such a phenomenon is important for the transport of tintinnids to the upper strata and may explain the maximum abundance of tintinnids in autumn. Moreover, on the surface, the water is usually over-saturated due to the exchanges with the atmosphere. Mixed water during the period of October to December may be the reason for the differences between the stations, especially in the $\mathrm{O}_{2}$ values $(\mathrm{V}>10 \%)$.

This study is the first to report on the composition and abundance of tintinnid species in the Büyükçekmece Bay, and the photographs of species have been illustrated (Figs. 5, 6). The abundance of tintinnids was negatively correlated with that of large phytoplankton species, which is probably due to their inability to consume these large prey. It is more likely that the tintinnids prey on nanoflagellates, but these were not included in this study and need to be explored in the future.

\section{ACKNOWLEDGMENTS}

The author is grateful to Assoc. Prof. Dr. Serhat Albayrak, Istanbul University, and Assoc. Prof. Dr. M. Ertan Çınar, Ege University, for their help with the statistical methods.

\section{REFERENCES}

Aubert, M., P. Revillon, J. Aubert, G. Leger, C. Drai, A. Arnoux and C. Diana. - 1990. Transfert de Pollutants entre la Mer Noire, la Mer de Marmara et la Mer Égée. Mers D’Europe. Etudes Hydrobiologiques, Chimiques et Biologiques, Tome 3, C.E.R.B.O.M. Nice.

Balech, E. - 1959. Tintinnoinea del Mediterráneo. Trab. Inst. esp. Oceanog., 28: 1-88.

Balech, E. - 1988. Los dinoflagelados del Atlántico sudoccidental. Publ. Espec. Inst. Esp. Oceanogr., 1: 223-310.

Balk1s, N. - 2000. Five dinoflagellate species new to Turkish seas. Oebalia., 26: 97-108.

Balkıs, N. - 2003. Seasonal variations in the phytoplankton and nutrient dynamics in the neritic water of the Büyükçekmece Bay, Sea of Marmara. J. Plank. Res., 25: 703-717.

Beşiktepe, S.T., H.I. Sur, E. Özsoy, M.A. Abdul Latif, T. Oğuz and Ü. Ünlüata. - 1995. The circulation and hydrography of the Marmara Sea. Prog. Oceanogr., 34: 285-334.

Bockstahler, K.R. and D.W. Coats. - 1993a. Spatial and temporal aspects of mixotrophy in Chesapeake Bay dinoflagellates. $J$. Euk. Microbiol., 40(1): 49-60.

Bockstahler, K.R. and D.W. Coats. - 1993b. Grazing of the mixotrophic dinoflagellate Gymnodinium sanguineum on ciliate populations of Chesapeake Bay. Mar. Biol., 116: 477-487.

Burkill, P.H., R.F.C. Mantoura, C.A. Llewellyn and N.J.P. Owens. - 1987. Microzooplankton grazing and selectivity of phytoplankton in coastal waters. Mar. Biol., 93: 581-590.

Capriulo, G. and D. Ninivaggi. - 1982. A comparison of the feeding activities of field collected tintinnids and copepods fed identical natural particle assemblages. Ann. Inst. Oceanogr., 
Paris, 58(S): 325-334

Coats, D.W. and N. Revelante. - 1999. Distributions and trophic implications of microzooplankton. Ecosystems at the land-sea margin: Drainage basin to coastal sea. Coastal and estuarine studies, 55. The American Geophysical Union.

Cupp, E.E. - 1943. Marine plankton diatoms of the west coast of North America. University of California Press, Berkeley.

Dodge, J.D. - 1982. Marine Dinoflagellates of the British Isles. Her Majesty's Stationery Office, London.

Dolan, J.R. and D.W. Coats. - 1990. Seasonal abundances of planktonic ciliates and microflagellates in mesohaline Chesapeake Bay waters. Est. Coast. Shelf Sci., 31: 157-175.

Drebes, G. - 1974. Marines phytoplankton. Eine Auswahl der Helgoländer Planktonalgen (Diatomeen, Peridineen) 151 Abbildungen, Georg Thieme Verlag Stuttgart.

Hallegraeff, G.M. - 1993. A review of harmful algal blooms and their apparent global increase. Phycological reviews 13. Phycologia, 32(2): 79-99.

Hansen, P.J. - 1989. The red-tide dinoflagellate Alexandrium tamarense: effects on behaviour and growth of a tintinnid ciliate. Mar. Ecol. Prog. Ser., 53: 105-116.

Hansen, P.J. - 1991a. Quantitative importance and trophic role of heterotrophic dinoflagellates in a coastal pelagial food web. Mar. Ecol. Prog. Ser., 73: 253-261.

Hansen, P.J. - 1991b. Dinophysis - a planktonic dinoflagellate genus which can act both as a prey and a predator of a ciliate. Mar. Ecol. Prog. Ser., 69: 201-204.

Hansen, P.J., A.D. Cembella and Q. Moestrup. - 1992. The marine dinoflagellate Alexandrium ostenfeldii: Paralytic shellfish toxin concentration, composition, and toxicity to a tintinnid ciliate. $J$. Phycol. 28: 597-603.

Hansen, P.J. and T.G. Nielsen. - 1997. Mixotrophic feeding of Fragilidium subglobosum (Dinophyceae) on three species of Ceratium: effects of prey concentration, prey species and light intensity. Mar. Ecol. Prog. Ser., 147: 187-196.

Hansen, P.J. - 1998. Phagotrophic mechanisms and prey selection in mixotrophic phytoflagellates. In: D.M. Anderson, A.D. Cembella and G.M. Hallegraeff (eds.), Physiological ecology of harmful algal blooms, pp. 525-537. NATO ASI Series, G41. Springer-Verlag Berlin Hiedelberg.

Hasle, G.R. - 1978. Concentrating Phytoplankton. The invertedmicroscope method. In: A. Sournia (ed.), Phytoplankton Manual, pp. 88-96. Monographs on oceanographic methodology, Paris, UNESCO.

Hasle, G.R. and E.E. Syvertsen. - 1997. Marine Diatoms. In: C.R. Tomas (ed.), Identifying marine phytoplankton. pp. 5-385. Academic Press a division of Harcourt Brace \& Company, San Diego, USA

Hendey, N.I. - 1964. An introductory account of the smaller algae of the British coastal waters. Part V: Bacillariophyceae (Diatoms). Fishery investigations, Ser. 4. Her Majesty's Stationery Office, London.

Ivanoff, A. - 1972. Introduction à l'océanographie. Vol. I. Librairie Vuibert, Paris.

Komakhidze, A. and N. Mazmanidi. - 1998. Black Sea biological diversity, Georgia. Black Sea Environmental Series, 8, United Nations Publications, New York.

Konsulov, A. - 1998. Black Sea biological diversity, Bulgaria. Black Sea Environmental Series, 5, United Nations Publications, New York.

Koray, T. and İ. Özel. - 1983. İzmir Körfezi planktonundan saptanan Tintinnoinea türleri. I. Ulusal Deniz ve Tatlisu Araştırmaları Kongresi Tebliğleri. 15-17 Ekim 1981, İzmir. E. U. F. F. Dergisi, Ser. B(I): 221-244.

Koray, T., B. Büyükışık, H. Parlak and Ş. Gökpınar. - 1992. İzmir Körfezi'nde deniz suyu kalitesini etkileyen tek hücreli organizmalar: Red-Tide ve diğer aşırı üreme olayları. Tr. J. Biol., 16: 135-157.

Koray, T., S. Gökpınar, L. Yurga, M. Turkoğlu and S. Polat. - 7th Apr. 1999. Microplankton species of Turkish Seas. <http://bornova.ege.edu.tr korayt/plankweb/chklists.html> (2002).

Lebour, M.V. - 1930. The planktonic diatoms of Northern Seas. Ray Soc., London.

Levinsen, H. and T.G. Nielsen. - 2002. The trophic role of marine pelagic ciliates and heterotrophic dinoflagellates in arctic and temperate coastal ecosystems: A cross-latitude comparison. Limnol. Oceanogr., 47(2): 427-439.

Marshall, S.M. - 1969. Order: Tintinnida. Conseil international pour l'explaration de la mer. Zooplankton sheet: 117-127.

Meriç, N. - 1986. Fishes encountered in Büyükçekmece Lake, Istanbul. I.Ü. Fen Fak. Mec. Ser. B(51): 41-46.

Meriç, N. - 1992. Büyükçekmece Baraj-Gölü balıkları üzerinde bir ön çalısma. Fırat Üniversitesi XI. Ulusal Biyoloji Kongresi, 24-

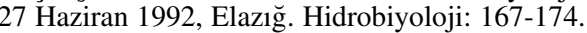

Öztürk, B. - 1999. Black Sea biological diversity, Turkey. Black Sea Environmental Series, 9, United Nations Publications, New York.

Paranjape, M.A. - 1990. Microzooplankton herbivory on the Grand Bank (New found land, Canada): a seasonal study. Mar. Biol., 107: 321-328.

Petranu, A. - 1997. Black Sea biological diversity, Romania. Black Sea Environmental Series, 4, United Nations Publications, New York.

Ricard, M. - 1987. Atlas du phytoplancton marin. Vol. 2: Diatomophyceés. Centre National de la Recherche Scientifique, Paris.

Sherr, E.B. and B.F. Sherr. - 1994. Bacterivory and herbivory: key roles of phagotrophic protists in pelagic food webs. Microb. Ecol., 28: 223-235.

Smalley, G.W. and D.W. Coats. - 2002. Ecology of the Red-Tide dinoflagellate Ceratium furca: distribution, mixotrophy and grazing impact on ciliate populations of Chesapeak Bay. $J$. Eukaryot. Microbiol., 49(1): 63-73.

Sorokin, Y.I., A.T. Tarkan, B. Öztürk and M. Albay. - 1995. Primary production, bacterioplankton and planktonic protozoa in the Marmara Sea. Turkish J. Mar. Sci., 1: 37-55.

Sournia, A. - 1968. Le genre Ceratium (Péridinien planctonique) dans le canal de Mozambique. Contribution a une révision mondiale. Vie milieu, sér. A, 18(2-3): 375-499.

Steidinger, K.A. and J. Williams. - 1970. Dinoflagellates. Memoirs of the Hourglass Cruises, Vol. 2, Florida Department of Natural Resources Marine Research Laboratory, St. Petersburg, Florida.

Steidinger, K.A. and K. Tangen. - 1997. Dinoflagellates. In: C.R. Tomas (ed.), Identifying marine phytoplankton. pp. 387-584. Academic Press a division of Harcourt Brace \& Company, San Diego, USA

Taylor, E.J.R. - 1976. Dinoflagellates from the international Indian Ocean expedition. A report on material collected by the "Anton Bruun" 1963-64, 132, Stuttgart.

Throndsen, J. - 1978. Preservation and storage. In: A. Sournia (ed.), Phytoplankton Manual, pp. 69-74. Monographs on oceanographic methodology, Paris, UNESCO.

Throndsen, J. - 1997. The planktonic marine flagellates. In: C.R. Tomas (ed.), Identifying marine phytoplankton. pp. 591-729. Academic Press, San Diego, USA.

Trégouboff, G. and M. Rose. - 1957. Manuel de Planctonologie Mediterranéenne, Tome I-II, Centre National de la Recherche Scientifique, Paris.

Tuğrul, S. and S.C. Polat. - 1995. Quantitative comparison of the influxes of nutrients and organic carbon into the Sea of Marmara both from anthropogenic sources and from the Black Sea. Pergamon, Wat. Sci. Tech., 32(2): 115-121.

Turner, J.T. and D.M. Anderson. - 1983. Zooplankton grazing during dinoflagellate blooms in a cape cod embayment, with observations of predation upon tintinnids by copepods. P.S.Z.N.I., Mar. Ecol., 4: 359-374.

Turner, J.T., P.A. Tester and P.J. Hansen. - 1998. Physiological ecology of harmful algal blooms. In: D.M. Anderson, A.D. Cembella and G.M. Hallegraeff (eds.), Interactions between toxic marine phytoplankton and metazoan and protistan grazers. pp. 453-474. NATO ASI series, G41.

Türkoğlu, M. and T. Koray. - 2000. Ecological and geographical distributions of the planktonic protista in the southern parts of the Black Sea (neritic waters of Sinop peninsula, Türkiye). E. $\ddot{U}$. Su Ürünleri dergisi, 17(1-2): 161-178.

Uysal, Z. - 1996. A net-plankton study in the Bosphorus junction of the Sea of Marmara. Tr. J. Botany, 20: 321-327.

Uysal, Z. and M. Ünsal. - 1996. Spatial distribution of net diatoms along adjacent water masses of different origin. Tr. J. Botany, 20: $519-525$.

Ünlüata, U., T. Oğuz, M.A. Latif and E. Özsoy. - 1990. On the physical oceanography of the Turkish Straits. In: L.J. Pratt (ed.), The physical oceanography of sea straits. pp. 25-60. Kluwer, Dortrecht.

Winkler, L.W. - 1888. The determination of dissolved oxygen in water. Berlin. Deut. Chem. Ges., 21: 2843-2855. 
Yüce, H. and A. Türker. - 1991. Marmara Denizi'nin fiziksel osinografik özellikleri ve Akdeniz suyunun Karadeniz'e girişi. Uluslararası çevre sorunları sempozyumu tebliğleri: 284-303.

Zaitsev, Y.P. and B.G. Alexandrov. - 1998. Black Sea biological diversity, Ukraine. Black Sea Environmental Series, 7, United Nations Publications, New York.
Zingone, A., M. Montresor and D. Marino. - 1990. Summer phytoplankton physiognomy in coastal waters of the Gulf of Naples. Mar. Ecol., 11(2): 157-172.

Scient. ed.: P. Jonsson 